\title{
La catálisis y fotocatálisis en la Facultad de Ciencias Químicas de la Universidad Autónoma de Nuevo León
}

\author{
Javier Rivera de la Rosa*
}

\begin{abstract}
RESUMEN: En esta contribución se describen las actividades de investigación, docencia y vinculación que se realizan en las áreas de catálisis y fotocatálisis en la Facultad de Ciencias Químicas (FCQ) de la Universidad Autónoma de Nuevo León (UANL). Se presentan los inicios de investigaciones en el área de catálisis y fotocatálisis en la FCQ de la UANL, que coincidió con la apertura de los posgrados de maestría y doctorado en ciencias con orientación en procesos sustentables, y la orientación en química analítica ambiental ambos en el Padrón Nacional de Posgrados de Calidad (PNPC) del Consejo Nacional de Ciencia y Tecnología (CONACyT) de México. Se cuenta con diferentes tipos de investigación en catálisis y fotocatálisis, tanto en la parte de síntesis y caracterización de nuevos catalizadores, estudios en diferentes tipos de reactores catalíticos para diferentes reacciones en aplicaciones ambientales y de biocombustibles. Las vinculaciones con grupos de catálisis con diferentes universidades de México y Estados Unidos de América, así como una doble titulación con la Universidad de Isla Baleares, en España. Se han dado servicios a la industria en área de catálisis y fotocatálisis y se tienen proyectos de colaboración con la plataforma solar de Almería, en España, por parte del grupo del Laboratorio de Fotocatálisis y Electroquímica Ambiental. En los posgrados relacionados con la investigación en catálisis se vislumbra un crecimiento a corto y mediano plazo en número de estudiantes del extranjero y productos académicos con colaboración internacional.
\end{abstract}

PALABRAS CLAVE: Cátalisis, fotocátalisis, reactores continuos, vinculación internacional.

ABSTRACT: This work describes the research, academic and collaboration activities carried out in the areas of catalysis and photocatalysis at the Faculty of Chemistry (FCQ, Spanish acronym) of the Autonomous University of Nuevo Leon (UANL, Spanish acronym). It presents the beginnings of research in the area of catalysis and photocatalysis in the FCQ of the UANL, coincided with the opening of the postgraduate Master and Doctorate in Sciences with a focus on Sustainable Processes and the focus in Environmental Analytical Chemistry in the National Register of Postgraduates Programs of Quality (PNPC, Spanish acronym) of the National Council of Science and Technology (CONACyT, Spanish acronym) of Mexico. There are different types of researches in catalysis and photocatalysis; with the synthesis and characterization of new catalysts, and studies in different configurations of catalytic reactors for different reactions in environmental and biofuels applications. The collaboration network with catalysis groups are in different universities in Mexico and the United States of America, as well as a double degree with the University of Balearic Island, in Spain. Technologic services have been provided to the industry in

Recibido: 23 de enero de 2016. Aceptado: 17 de marzo de 2017.

*Universidad Autónoma de Nuevo León, UANL, Facultad de Ciencias Químicas, Ave. Universidad s/N, Cd. Universitaria, C.P. 64451, San Nicolás de los Garza, Nuevo León, México. Correspondencia: (javier.riverad@uanl.edu.mx).

El doctor Javier Rivera de la Rosa recibió su grado de Dottore in Ricerca in Ingegneria Chimica del Politecnico di Torino, Italia en el 2003. Es maestro en ciencias en ingeniería mecánica con especialidad en materiales por la UANL e ingeniero químico también por la UANL. 
Mundo Nano | CATÁLISIS EN MÉXICo | www.mundonano.unam.mx

10(18), enero-junio 2017 | DOI: 10.22201/ceiich.24485691e.2017.18.60083

catalysis and photocatalysis and currently there is a project of collaboration with the solar platform of Almeria in Spain. In the postgraduate studies of catalysis research shows a growth in the short and medium term in the number of students with academic products with international collaboration.

KEYWORDS: Catalysis, photocatalysis, continues reactors, international collaboration.

\section{Introducción}

La Facultad de Ciencias Químicas (FCQ) de la Universidad Autónoma de Nuevo León (UANL) fue fundada en 1933, siendo su precedente la Escuela de Química y Farmacia en 1931, actualmente se ofrecen cuatro programas de licenciatura: químico farmacéutico biólogo, licenciado en química industrial, ingeniero químico, e, ingeniero industrial administrador. Todos los programas acreditados a nivel nacional por organismos reconocidos como el Consejo de Acreditación de la Enseñanza de la Ingeniería Superior, A. C. (CACEI), Consejo Nacional de Enseñanza y del Ejercicio Profesional de las de Ciencias Químicas, A. C. (CONAECQ) y el Consejo Mexicano para la Acreditación de la Educación Farmacéutica, A. C. (COMAEF), también reconocidos por organismos extranjeros como The Royal Society of Chemestry y actualmente se ha sometido bajo la visita de evaluación los programas de ingeniería de parte del American Board of Engineering and Technology (ABET) de los Estados Unidos de América.

La oferta de estudios de posgrado en la FCQ de la UANL son: maestría en administración con seis orientaciones, maestría en ingeniería industrial con tres orientaciones, cinco maestrías en ciencias con diferentes orientaciones: en procesos sustentables, farmacia, química analítica ambiental, química de materiales, y, microbiología aplicada así como también cinco doctorados en ciencias con las mismas orientaciones anteriormente mencionadas; estos últimos diez programas se encuentran dentro el Padrón Nacional de Posgrados de Calidad (PNPC) del Consejo Nacional de Ciencia y Tecnología (CONACyT) de México, y muy recientemente se ofrece la especialidad en sustentabilidad de los procesos del petróleo.

La FCQ de la UANL cuenta actualmente con cerca de 4,500 estudiantes de pregrado y 500 de posgrado, así como también 156 profesores de tiempo completo de los cuales 68 pertenecen al Sistema Nacional de Investigadores. La FCQ cuenta con dos campus, uno ubicado en Ciudad Universitaria en el municipio de San Nicolás de los Garza y una División de Estudios Superiores (DES) en la colonia Treviño en el municipio de Monterrey. Dentro de sus instalaciones cuenta con cerca de 40 laboratorios de enseñanza y de investigación, además cuenta con una Subdirección de Servicios Tecnológicos donde se brindan diferente tipos de venta de servicios y productos a la industria, con Laboratorio de Análisis Clínico, Laboratorio de Microbiología, Laboratorio de Alimentos, Laboratorio de Pruebas e Investigación de Cerámica, Centro de Negocios y Educación Continua (CNyEN) y particularmente el Laboratorio de Servicios Profesionales, el cual cuenta con las siguientes acredi- 
taciones: Aguas Residuales, AG-051-008/10, Fisicoquímico, A-064-009/10, Residuos R-0150-50-015/10, y muy próximamente con la apertura del Laboratorio de Sustentabilidad de los Procesos del Petróleo. A través del CNyeN los profesores investigadores de tiempo completo de la FCQ han desarrollado proyectos de vinculación con la industria local y nacional. También se ofrecen cursos de educación continua con cursos cortos y diplomados en varios temas como calidad, artículos de cosmetología, seguridad industrial entre otros. Para la realización de las actividades se cuenta con un sistema de clasificación, recolección y confinamiento de residuos peligrosos y municipales producidos en todos sus laboratorios y actividades de toda la comunidad [1], cabe denotar que la FCQ de la UANL ha obtenido el certificado de Calidad Ambiental Nivel 1 de parte de la Procuraduría Federal de Protección al Ambiente (PROFEPA) los años 2012, 2014 y 2016. Esta evaluación se realizó durante una auditoría para el control de las cargas ambientales de agua, aire, suelo y subsuelo, residuos peligrosos, residuos no peligrosos, riesgos y administración ambientales. También es importante mencionar que los procedimientos académicos, tecnológicos y trámites administrativos están bajo la norma ISO 9001:2008.

Dentro de este entorno de docencia, servicios a la industria y gestión de los residuos peligrosos y no peligrosos, con procedimientos bajo un sistema de calidad, los investigadores de las áreas de catálisis y fotocatálisis de la FCQ de la UANL gestionan recursos externos, dirigen proyectos, realizan vinculación y varios de ellos son evaluadores en el PNPC del CONACyT, en los organismos nacionales mencionados y procuran la vinculación con el sector privado y académico nacional e internacional. Este trabajo describe los programas de posgrados donde se desempeñan investigaciones en catálisis y fotocatálisis, infraestructura disponible y vinculaciones de los investigadores actuales de la FCQ de la UANL, así como una visión a corto y mediano plazo.

\section{Posgrados relacionados con catálisis y fotocatálisis}

Los posgrados relacionados con catálisis y fotocatálisis en la FCQ de la UANL son maestría en ciencias con orientación en procesos sustentables, maestría en ciencias con orientación en química analítica ambiental, doctorado en ciencias con orientación en procesos sustentables, y, doctorado en ciencias con orientación en química analítica ambiental. Ambos programas en el PNPC del CONACyT con el nivel de consolidados y próximamente en el 2017 se aplicará para el nivel de internacional. Los Cuerpos Académicos organizados que principalmente realizan productos académicos y de vinculación dentro de estos posgrados son el de Cuerpo Académico de Ingeniería Química y el Cuerpo Académico de Evaluación y Tratamiento de Contaminantes Ambientales, ambos consolidados según el Programa para el Desarrollo Profesional Docente (PRODEP). Desde el 2006 se han producidos en tiempo y forma varias tesis de maestría y doctorado en ciencias en ambas orientaciones, obteniendo reco- 
nocimientos nacionales e internacionales como mejor tesis de ingeniería y tecnología de la UANL, premios de investigación en la áreas de ciencias, así como en ingeniería y tecnología de la UANL, premio de mejor tesis de maestría en catálisis de parte de la Academia de Catálisis A.C. (ACAT) de México, reconocimiento de Beca Kokes por parte de la North American Catalysis Society para asistir al North American Meeting. De las tesis producidas se han derivado presentaciones en congresos nacionales e internacionales tales como en el Encuentro Nacional de la Academia Mexicana de Investigación y Docencia en Ingeniería Química (AMIDIQ), Congreso Mexicano de Catálisis (CMC), Congreso Mexicano de Química (CMQ), Congreso Internacional de la North American Catalysis Society, entre otros. También se ha publicado en varias revistas científicas indizadas de acuerdo con la lista del Science Citation Index Report como: Applied Catalysis B. Enviromental, Applied Catalysis A. General, The Chemical Engineering Journal, Journal of Hazardous Materials, Journal of Materials Science, entre otras, los artículos a los que hacemos mención se encuentran en el listado de referencias.

Las Líneas de Generación y Aplicación del Conocimiento (LGAC) en las cuales se desarrollan los proyectos y los profesores investigadores colaboran entre sí son: Aplicación de métodos analíticos en el desarrollo de procesos para tratamiento de contaminantes en los posgrados con orientación en química analítica ambiental y la LGAC de procesos sustentables en los posgrados con orientación en procesos sustentables.

La maestría en ciencias y doctorado en ciencias con orientación en química analítica ambiental cuenta con un convenio de doble titulación con la Universidad de Islas Baleares (UIB) en España, actualmente han graduado a un doctor en ciencias, y se han realizado estancias de investigación de profesores y estudiantes del programa de la FCQ de la UANL en esa universidad de España.

Los proyectos relacionados con catálisis y fotocatálisis de los cuatro programas en ambas orientaciones se pueden describir en los tres rubros de síntesis, caracterización y aplicaciones.

\section{Sintesis}

Síntesis de óxidos metálicos como dióxido de titanio, alúmina y materiales micro y mesoporosos como la SBA-15 y zeolitas como soportes principalmente (aunque tiene contribución en la actividad catalítica y fotocatalítica) que pueden ser dopados con metales de transición como Fe, Zn, La, Zr, Mn, $\mathrm{Cu}$, entre otros, y por lo tanto se ven modificadas sus propiedades texturales (de los soportes) y a su vez en su actividad catalítica. También se está experimentado actualmente con dopaje con aniones como el fluoruro y nitrógeno. Otra modalidad es utilizar los soportes depositando nanopartículas de metales como Pd, Pt y Fe elemental a bajas concentraciones y a su vez buscar nanopartículas de aleaciones de la combinación de Pd-Fe y Pt-Fe los cuales dan como resultado nuevos sitios activos. Generalmente los catalizadores 
(soportes dopado o puros) han sido sintetizados por técnicas como sol-gel o coprecitación, las cuales los profesores investigadores en catálisis y fotocatálisis tienen bien dominadas, y cuentan con una amplia experiencia para proponer modificaciones a la técnica (sobre todo sol-gel) y provocar nuevos e interesantes sitios activos. También se han utilizado soportes comerciales (comprados de distribuidor comercial) como alúmina y zeolitas. Actualmente se estan sintetizando zeolitas de tamaño nanométrico, como soportes.

\section{Caracterización}

Los catalizadores y fotocatalizadores sintetizados para los proyectos de investigación en los laboratorios de la FCQ de la UANL, son caracterizados en fases intermedias de preparación (para control y conocimiento del método), frescos preparados e inclusive ya usados en reacción. Las técnicas para la caracterización son múltiples entre la cuales se pueden mencionar: análisis térmicos diferencial, gravimetría y calorimetría diferencial de barrido, espectrometría de infrarrojo por transformada de Fourier (FT-IR), espectrometría de UV y visible para sólidos y líquidos, difracción de rayos $\mathrm{X}$ de polvo, fisisorción de nitrógeno, microscopia electrónica de barrido (SEM, por sus siglas en inglés), análisis elemental por diferentes técnicas analíticas. En otros laboratorios de la UANL también se tiene acceso a técnicas como microscopía electrónica de transmisión de alta resolución (HRTEM por sus siglas en inglés), espectroscopía fotoelectrónica de rayos X (XPS por sus siglas en inglés). Asimismo, se cuenta con caracterización especializada resonancia magnética nuclear (NMR, por sus siglas en inglés) para sólidos con colaboración de las Universidades de The City University of New York y Clemson University en Estados Unidos de América.

\section{Aplicaciones (reactores)}

Las aplicaciones de los catalizadores y fotocatalizadores sintetizados son del tipo ambiental, industrial y producción de biocombustibles. Las reacciones son en estado líquido y gaseoso, en estado continuo o reactor tipo carga y descarga (batch). Se han construido diferentes tipos de reactores continuos para estudiar la cinética y efectos de transferencia de masa para las reacciones de interés. Los reactores diseñados, construidos y usados han sido de tipo cama empacada, monolitos, placas paralelas, reactor anular y también bifásico de las fases fluidas. Los reactores cuentan con medidores de presión y temperatura y los datos son recolectados por interfases en equipo de cómputo. El análisis de concentración de los reactivos y productos de reacción son a través de técnicas como cromatografía de gases (GC), cromatografía de alta eficiencia de líquido (HPLC, por sus siglas en inglés) y UV-vis de soluciones líquidas. Se determinan cinéticas y se analizan y los efectos de transferencia de masa son evaluados. 

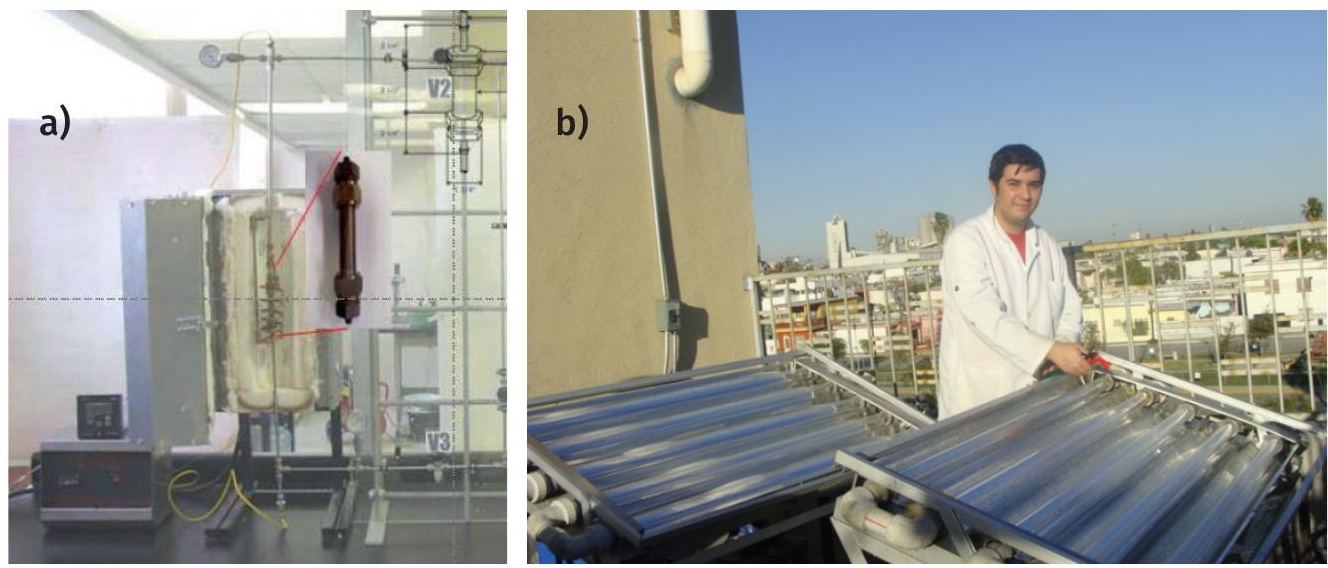

FIGURA1. a) Reactor continuo de cama empacada con medidores de presión y temperatura; b) reactor solar de tipo captador parabólico compuesto (CPC).

\section{Infraestructura}

En la FCQ de la UANL se han adquirido diferentes equipos para la investigación por diferentes fuentes de financiamiento, por convocatorias del CONACYT, PRODEP, proyectos con la industria y otras fuentes externas. A continuación se enlistan algunos equipos convenientes para la investigación en catálisis y fotocatálisis:

- Equipo de fisisorción de nitrógeno Autosorb-1 (Quantachrome Co.).

- Cromatógrafos de gases de diferentes modelos 5890, 6890 y 7890 A de Agilent y con diferentes detectores como FID y TCD y de masas.

- Cromatógrafos de líquidos de alta eficiencia (HPLC) con diferentes detectores como IR, arreglos de diodos y masas.

- Analizador elemental por espectrometría de plasma por microondas Agilent 4200.

- Difractómetro de rayos-X, modelo D2 Phaser, Bruker.

- Espectrómetro UV-vis, modelo 300, Nicolet con accesorio de reflectancia difusa.

- Espectrómetro FTIR, modelo 200-X Interspec con accesorio de reflectancia atenuada.

- Calorímetro de barrido diferencial DSC, modelo DSC-50, Shimadzu.

- Analizador térmico diferencial DTA, modelo DTA-50, Shimadzu.

- Viscosímetro, modelo MV-3000, Cambridge Intruments.

- Densímetro, Anton Par.

- Microscopio de barrido electrónico SEM, modelo Nescope 6000, JEOL.

- Microscopio estereoscópico, modelo MV-439, National Instruments. 
junto y asesorías de tesis tanto de la UANL como de la Universidad de Almería con la cual está vinculada la PSA.

\section{Principales logros en catálisis y fotocatálisis}

Los principales logros de la FCQ de la UANL en catálisis y fotocatálisis se pueden resumir en cuatro puntos:

1. Formación de cuerpos académicos consolidados en la investigación en catálisis y fotocatálisis. Debido al número de estudiantes locales, nacionales y extranjeros (actualmente de Colombia y Venezuela), a los productos de tesis en tiempo y forma y los artículos en revistas de alto impacto del área de catálisis. Vinculaciones efectivas y estancias de los estudiantes de los posgrados mencionados en diferentes institutos de investigación y universidades nacionales e internacionales.

2. Disponibilidad de infraestructura que se ha obtenido a través de fondos de convocatorias del CONACyT y con proyectos con la industria. Los estudiantes de posgrado y licenciatura que desean hacer su tesis en los campos de catálisis y fotocatálisis encuentran en la FCQ y en toda la UANL una completa línea de diferentes técnicas experimentales de medición de diferentes propiedades, y las vinculaciones nacionales e internacionales permite tener acceso a equipo especializado, la espectrometría de NMR en estado sólido con polarización cruzada y resonancia magnética nuclear con giro al ángulo mágico (magic angle spinning), permite obtener artículos de alta calidad.

3. La doble titulación que pueden aplicar los estudiantes de posgrado de la maestría en ciencias y doctorado en ciencias con orientación en química analítica ambiental con UIB en España, es un logro ejemplar que se está replicando en otras orientaciones de otros posgrados de la FCQ de la UANL.

4. El impacto nacional e internacional de los Cuerpos Académicos Consolidados de la FCQ de la UANL a través de sus productos académicos y de vinculación les da la pertinencia de organizar el próximo Congreso Mexicano de Catálisis XV Nacional y VI Internacional [7].

\section{Visión al futuro de la investigación en catálisis y fotocatálisis}

La investigación en catálisis y fotocatálisis en la FCQ de la UANL tiene como perspectivas el crecimiento en todos los rubros; conocimiento, productos de alto impacto a nivel internacional, consolidar las vinculaciones actuales con las universidades nacionales e internacionales, replicar el modelo de doble titulación en las demás orientaciones de los posgrados en ciencias donde se 
desarrolla investigación en catálisis y fotocatálisis. Lograr otros convenios de colaboración y doble titulación con otras universidades en el extranjero.

La investigación de catálisis y fotocatálisis en la FCQ de la UANL desea crear una escuela en este ramo de la investigación a través de sus egresados de doctorado para luego ir a otras instituciones nacionales o internacionales a replicar el modelo aprendido en metodología de la investigación en catálisis y fotocatálisis.

La vinculación con el sector privado y público tiene un amplio rango de oportunidad y esto se logrará con los proyectos en conjunto con estos organismos.

Los posgrados de la FCQ de la UANL relacionados con catálisis y fotocatálisis serán una referencia internacional de calidad e impacto en la investigación en este ramo, y por lo tanto estudiantes nacionales y extranjeros desearán realizar estancias o estudios completos en ellos. Más profesores y posdoctorados erigirán a la FCQ de la UANL para realizar estancias en el ramo de investigación de catálisis y fotocatálisis.

\section{Sitios de interés}

- Página institucional:

<http://www.fcq.uanl.mx/>

\section{Referencias}

[1]. E. R. Lara, J. R. de la Rosa, A. I. R. Castillo, F. J. Cerino-Córdova, U. J. L. Chuken, S. S. F. Delgadillo, P. Rivas-García. (2017). A comprehensive hazardous waste management program in a Chemistry School at a Mexican university. Journal of Cleaner Production, vol 142, 1486, 1491.

[2]. Daniela Xulú Martínez-Vargas, Javier Rivera de la Rosa, Carlos J. Lucio-Ortiz, Aracely Hernández Ramírez, Gerardo A. Flores-Escamilla, Carlos D. García. (2015). Photocatalytic degradation of trichloroethylene in a continuous annular reactor using $\mathrm{Cu}$-doped $\mathrm{TiO}_{2}$ catalysts by sol-gel synthesis. Applied Catalysis B: Environmental, vol. 179, 249-261.

[3]. Daniela X. Martínez Vargas, Javier Rivera de La Rosa, Saba Arif Iyoob, Carlos J. Lucio Ortiz, Felipe J. Cerino Córdova, Carlos D. García. (2015). Phenol oxidation by air using a Co (II) Salen complex catalyst supported on nanoporous materials: Synthesis, characterization and kinetic analysis. Applied catalysis A., vol. 179, 249-261.

[4]. M. Jiménez-Tototzintle, I. Oller, A. Hernández-Ramírez, M. I. Maldonado. (2015). Remediation of agro-food industry effluents by biotreatment combined with supported $\mathrm{TiO}_{2} / \mathrm{H}_{2} \mathrm{O}_{2}$ solar photocatalysis. The Chemical Engineering Journal, vol. 273, 205-213.

[5]. Benjamín Garza-Campos, Enric Brillas, Aracely Hernández-Ramírez, Edgar J. Ruiz-Ruiz. (2016). Salicylic acid degradation by advanced oxidation processes. 
Mundo Nano | CATÁLISIS EN MÉXICo | www.mundonano.unam.mx

10(18), enero-junio 2017 | DOI: 10.22201/ceiich.24485691e.2017.18.60083

Coupling of solar photoelectro-Fenton and solar heterogeneous photocatalysis. Journal of hazardous materials, vol. 319, 34-42.

[6]. Iliana Medina-Ramírez, Jingbo Louise Liu, Araceli Hernández-Ramírez, Miguel A. Gracia-Pinilla. (2014). Synthesis, characterization, photocatalytic evaluation, and toxicity studies of $\mathrm{TiO}_{2}-\mathrm{Fe}_{3}$ + nanocatalyst. Journal of Materials Science, vol. 49, 5309-5323.

[7]. XV Congreso Mexicano de Catálisis y VI Internacional. (2017). <http://eventos. uanl.mx/cmc_2017/index.html>. 